\title{
Esplenectomía laparoscópica en esplenomegalia masiva por linfoma no Hodgkin de zona marginal
}

Massive laparoscopic splenectomy for marginal zone non-Hodgkin lymphoma Esplenectomia laparoscópica em esplenomegalia maciça devido a linfoma não-Hodgkin de zona marginal

\author{
Martina Tristant , Daniel González González²
}

\section{Resumen}

Introducción: si bien la esplenectomía laparoscópica en esplenomegalias masivas y supramasivas constituye un desafío técnico, su realización es factible y segura en centros con equipos con experiencia en cirugía laparoscópica.

Objetivo: presentar el primer caso de esplenectomía laparoscópica en esplenomegalia masiva realizada en Uruguay.

Caso clínico: se trata de una paciente de 70 años portadora de una pancitopenia periférica, esplenomegalia masiva y diagnóstico realizado por punción de médula ósea de neoplasia linfoproliferativa tipo B de bajo grado, a quien se le indicó la esplenectomía con fines diagnósticos y terapéuticos. La paciente se operó en decúbito lateral derecho a 15 grados, los trócares se colocaron bajo visión directa adaptados al tamaño del bazo que se extendía desde el diafragma hasta el estrecho superior de la pelvis. Se realizó la esplenectomía en un tiempo de 220 minutos, extrayéndose la pieza íntegra y sin haberla colocado en bolsa a través de un hemi Pfannenstiel, protegiendo la pared con un retractor de heridas quirúrgicas. No presentó complicaciones, fue dada de alta a las 48 horas. El hemograma realizado a las 24 horas demostró un aumento de las cifras de todas las series celulares y el informe anatomopatológico diagnosticó un linfoma no Hodgkin de zona marginal.

Discusión: la esplenectomía laparoscópica en esplenomegalias masivas requiere de un mayor tiempo quirúrgico, aunque las pérdidas sanguíneas y la estadía hospitalaria son menores en comparación a los procedimientos convencionales, presentando una morbilidad similar. En la experiencia inicial de los equipos quirúrgicos se reporta un porcentaje de conversiones y reingresos cercanos al $30 \%$.

Palabras clave: Esplenomegalia masiva

Esplenectomía

Laparoscopía

Linfoma no Hodgkin

Key words: Massive splenomegaly

Splenectomy

Laparoscopy

Lymphoma, non-Hodgkin

\footnotetext{
1. Residente de Cirugía General.

2. Profesor Titular de Clínica Quirúrgica. Jefe del Departamento de Cirugía de la Cooperativa Médica de Florida.

Clínica Quirúrgica 3. Facultad de Medicina. Universidad de la República. Montevideo. Uruguay.

Correspondencia: Martina Tristant. Correo electrónico: tristant.martina@gmail.com.

Recibido: 4/2/2021

Aprobado: 25/5/2021

Attribution-NonCommercial 4.0 International (CC BY-NC 4.0)
} 


\section{Introducción}

La esplenectomía laparoscópica se ha impuesto en el tratamiento electivo de diversas enfermedades esplénicas. Sin embargo, el tamaño del bazo ha sido considerado como contraindicación relativa, siendo un procedimiento no recomendado para esplenomegalias masivas por las guías europeas del año $2008^{(1)}$.

Una solución para poder reunir las ventajas de los procedimientos laparoscópicos y la seguridad en este tipo de casos fue la realización de la esplenectomía laparoscópica

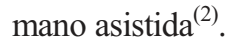

Otra alternativa es la embolización de la arteria esplénica preoperatoria para reducir el tamaño del bazo, hecho que facilita el procedimiento, reduciendo las conversiones y sangrados intraoperatorios ${ }^{(3)}$.

De todas formas, la adquisición de experiencia de diversos grupos en procedimientos laparoscópicos ha llevado a su realización exclusivamente por vía laparoscópica con resultados favorables ${ }^{(4-7)}$.

La definición de esplenemegalia masiva y supermasiva o gigante se basa en la longitud craneocaudal del bazo o su peso. Las primeras son aquellas que superan los $17 \mathrm{~cm}$ según $\mathrm{Grahn}^{(4)}$ o $20 \mathrm{~cm}$ según Swanson ${ }^{(2)}$, o un peso superior a 600 g. Las segundas superan los $22 \mathrm{~cm} \mathrm{o} 1.600 \mathrm{~g}$.

El objetivo del presente trabajo es comunicar el primer caso de esplenectomía laparoscópica en una esplenomegalia masiva realizada en Uruguay.

\section{Caso clínico}

Paciente de 70 años, sexo femenino, con antecedentes de cáncer de cuello uterino tratada con radioterapia, tiroidectomía total por cáncer papilar de tiroides, colecistectomía por colecistitis aguda por abordaje convencional, resección anterior de recto videoasistida por cáncer de la unión recto sigmoidea.

En estudio por pancitopenia periférica se realizó biopsia de médula ósea que informó neoplasia linfoproliferativa $\mathrm{B}$ de bajo grado.

Se inició tratamiento en base a eritropoyetina, corticoides y vitaminoterapia, mejorando las cifras de glóbulos blancos y rojos, permaneciendo con plaquetopenia con valores de 32.000 elementos $/ \mathrm{mm}^{3}$.

En base a ello se decidió la realización de la esplenectomía con fines diagnósticos y terapéuticos.

Al examen: paciente adelgazada, mucosas hipocoloreadas. No se palpan adenomegalias superficiales.

Abdomen plano, con cicatriz transversa de hipocondrio derecho y Pfannenstiel (por donde se extrajo la pieza de colectomía) continentes. Incisiones de colocación de trócares de la colectomía en buenas condiciones.

A la palpación presenta esplenomegalia grado 4 .

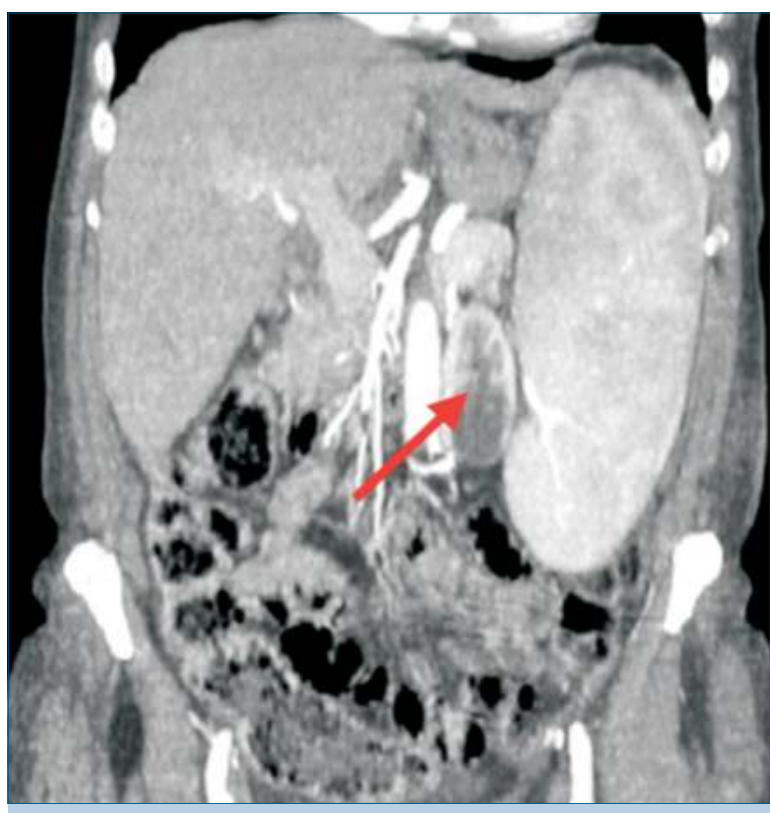

Figura 1. Tomografía abdominal de abdomen y pelvis. Se aprecia el bazo extendido desde la cúpula diafragmática izquierda hasta el estrecho superior de la pelvis, desplazando el riñón izquierdo hacia la línea media (flecha roja).

Se realizó tomografía tóraco-abdómino-pélvica que informó: esplenomegalia de densidad homogénea de $20 \mathrm{~cm}$ de longitud. Riñón izquierdo desplazado medialmente por la esplenomegalia. No se evidenciaron adenomegalias (figura 1).

Hemograma preoperatorio: 3.630 glóbulos blan$\cos / \mathrm{mm}^{3}, 3.310 .000$ glóbulos rojos $/ \mathrm{mm}^{3}, 25.900$ plaque$\operatorname{tas} / \mathrm{mm}^{3}$.

Se realizó cirugía de coordinación con paciente en posición de leve decúbito lateral derecho a 15 grados y cambrado. Se colocaron 4 trócares bajo visión directa y adaptados al tamaño del bazo, 2 de $10 \mathrm{~mm}$, uno en la línea media entre el ombligo y reborde costal izquierdo por donde se colocó la óptica y otro en flanco izquierdo, 2 de $5 \mathrm{~mm}$, uno en epigastrio y otro en flanco izquierdo, por debajo y por fuera del anterior (figura 2).

De la exploración se destacó un bazo cuyo polo superior se topografía en el hemidiafragma izquierdo y su polo inferior en la pelvis (figura 3 ) y un bazo accesorio sobre el pedículo esplénico (figura 4). Luego del descenso del ángulo izquierdo del colon se abordó la cara interna del bazo desde el polo inferior con la utilización de un sellador de vasos, realizando la esplenectomía del bazo accesorio que se extrajo íntegro en bolsa por uno de los abordajes de flanco izquierdo. Se utilizaron clips de 400 para la ligadura y sección del pedículo esplénico. Se completó la liberación del bazo por su cara externa. 


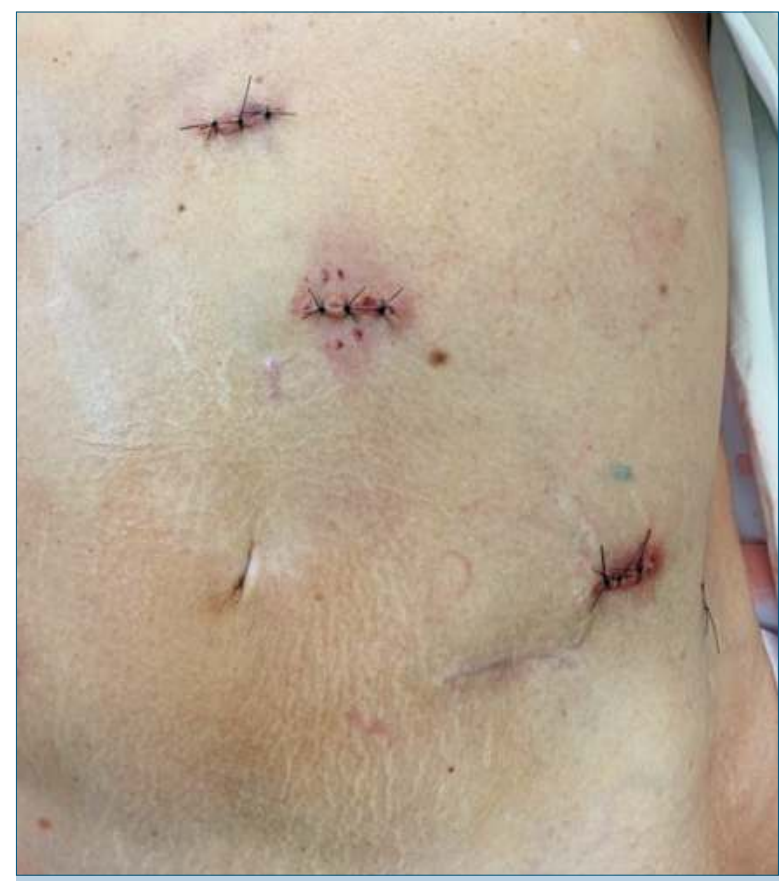

Figura 2. Vista de las incisiones para la colocación de los trócares y de la extracción de la pieza.

La extracción del bazo íntegro (figura 5) se realizó mediante una incisión emplazada en la mitad izquierda del un Pfannenstiel previo (figura 2) y la colocación de un retractor y protector de heridas quirúrgicas mediano. Al ingresar en la cavidad peritoneal el polo inferior del bazo se encontraba sobre la cicatriz y con la utilización de una pinza de Foester se completó la extracción.

La paciente presentó buena evolución postoperatoria, con escaso dolor, comenzó a tolerar vía oral a las 6 horas, deambular a las 12 horas y fue dada de alta a las 48 horas.

El hemograma de control realizado a las 24 horas evidenció un ascenso de las plaquetas: $82.000 / \mathrm{mm}^{3}, 27.000$ glóbulos blancos $/ \mathrm{mm}^{3}$ y 3.750 .000 glóbulos rojos $/ \mathrm{mm}^{3}$.

El estudio macroscópico de la pieza informó: bazo de $655 \mathrm{~g}$, cuyas dimensiones fueron $203 \mathrm{~mm}$ por $130 \mathrm{~mm}$ por $50 \mathrm{~mm}$ (figura 5). Bazo accesorio de $21 \mathrm{~mm}$ por $13 \mathrm{~mm}$ por $10 \mathrm{~mm}$.

El informe del estudio microscópico estableció: parénquima esplénico cuyas características morfológicas e inmunofenotipos son compatibles con un linfoma no Hodgkin de zona marginal (figura 6).

\section{Discusión}

La esplenectomía laparoscópica en esplenomegalias masivas o supramasivas constituye un desafío técnico, aunque es factible su realización en forma segura y con buenos resultados según lo demostrado por Grahn ${ }^{(4)}$, Casaccia $^{(5)}$, Tsamalaidza ${ }^{(6)}$ y Rodríguez-Luna ${ }^{(8)}$.

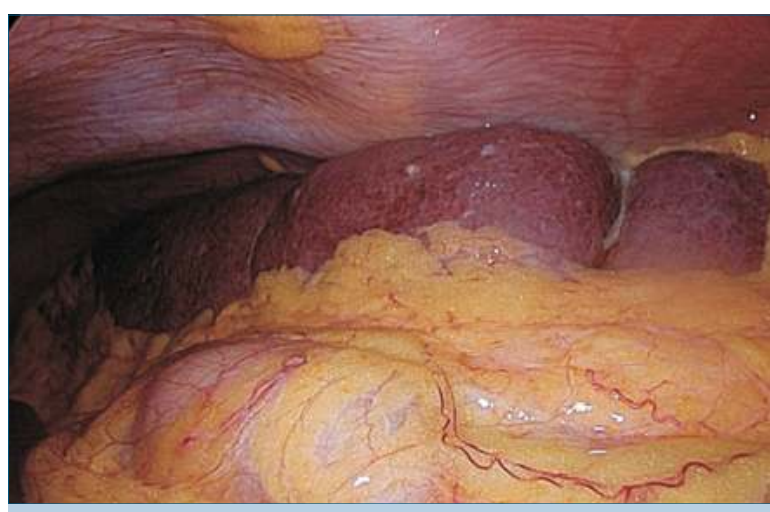

Figura 3. Vista intraoperatoria del bazo ocupando el hemiabdomen izquierdo.

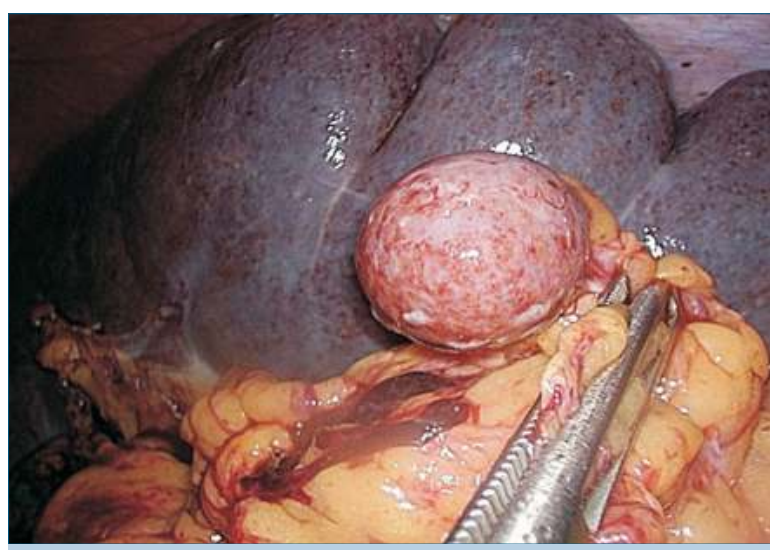

Figura 4. Vista intraoperatoria de bazo accesorio

Casaccia ${ }^{(5)}$ a través de la comparación de 2 grupos de pacientes con esplenomegalias masivas, uno sometido a cirugía laparoscópica (24 casos) y otro a cirugía convencional (31 casos) comunicó un mayor tiempo operatorio para el grupo de cirugía laparoscópica, pero menor pérdida sanguínea y estadía hospitalaria. No presentó conversiones y la morbilidad fue similar en ambos grupos.

Tsamalaidza ${ }^{(6)}$ arribó a la misma conclusión habiendo comparado un grupo de 27 pacientes por vía laparoscópica, 12 por abordaje mano asistido y 47 convencional. En este caso sí reportó conversiones: 3 por vía laparoscópica y 1 por abordaje mano asistido, siendo los motivos el tamaño del bazo, dificultades técnicas y sangrado.

$\mathrm{Grahn}^{(4)}$ en su serie de 25 casos comunicó un primer período con 33\% de conversiones, sin embargo en el último, que abarcó 2 años, no hubo conversiones. Este autor concluye que la experiencia adquirida por el grupo y el centro asistencial ha llevado a la mejora de los resultados ya que además de la reducción de las conversiones, no hubo reoperaciones por sangrados, tampoco mortalidad y la estadía hospitalaria fue de 3,8 días, en comparación a los 9 días para la esplenectomía por vía convencional. 


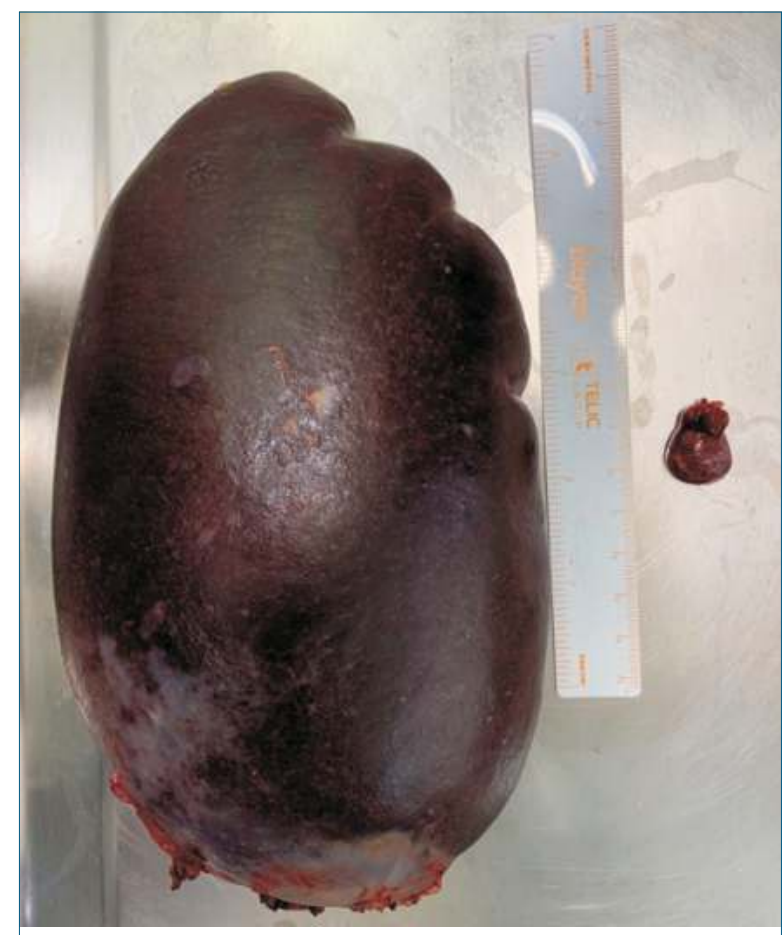

Figura 5. Imagen de la pieza operatoria: bazo y bazo accesorio.

Sin embargo, cuando se comparan esplenectomías realizadas sobre bazos de tamaño normal y aquellos con esplenomegalias masivas sí existen diferencias, una de ellas lógica como puede ser el tiempo operatorio (112 minutos para los bazos de tamaño normal y 171 para las esplenomegalias masivas) y en nuestro caso superior, 220 minutos, ya que el objetivo es evitar un sangrado por desgarro parenquimatoso o lesión vascular del pedículo o sus ramas que obliguen a la conversión y por lo tanto lo más importante sobre todo cuando se trata de los primeros casos realizados es la minuciosidad en los diversos tiempos operatorios. La otra diferencia son los reingresos que también resultaron ser superiores para las esplenomegalias masivas ( $27 \%$ vs $6 \%)^{(9)}$.

A modo de síntesis Rodríguez-Luna ${ }^{(8)}$ en un metaanálisis publicado en 2021 que incluyó 20 trabajos que comparan las esplenectomías laparoscópicas exclusivas, mano asistidas y abiertas en esplenomegalias masivas concluyó que la esplenectomía laparoscópica es factible y segura.

Otros aspectos técnicos que requieren ser considerados son la posición del paciente para la cirugía y la extracción del bazo.

En cuanto a la posición, es habitual utilizar el decúbito lateral derecho a 45 grados para aquellos bazos de tamaño normal, con el objetivo de que el mismo quede colgando de la pared del abdomen y así exponer el hilio. Sin embargo, en esplenomegalias esta posición determina el ocultamien-

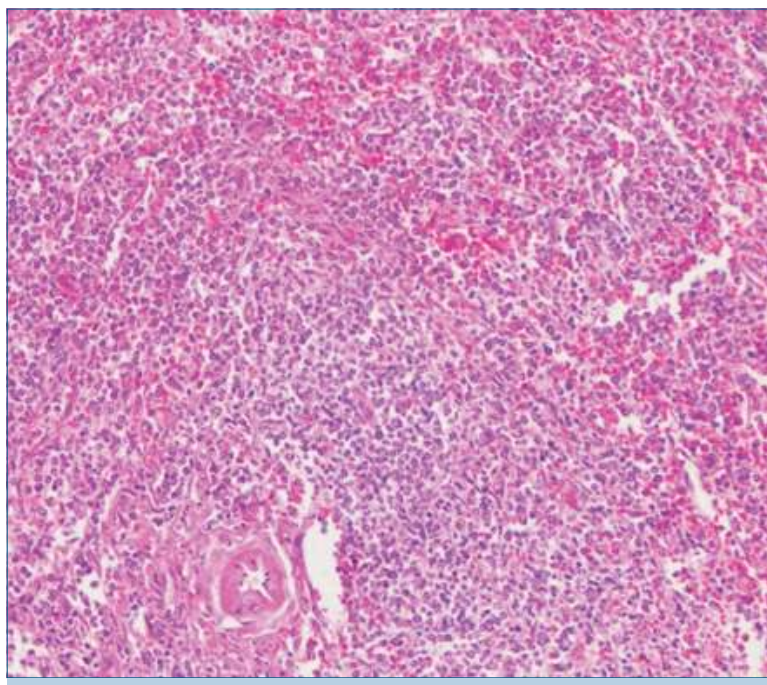

Figura 6. Imagen del estudio microscópico de la pieza teñida con hematoxilina-eosina y un aumento de $100 \mathrm{x}$.

to del hilio por la caída que se produce del bazo sobre él. Por tanto un ligero decúbito (10-15 grados) como el que empleamos o incluso el decúbito dorsal son los más adecuados $^{(4)}$.

En cuanto a la extracción del bazo es clásico su colocación en una bolsa, posterior maceración y extracción ya sea a través de una de las incisiones utilizadas para colocar alguno de los trocares u otra incisión complementaria como puede ser un Pfannenstiel ${ }^{(7)}$. Todo ello constituye una segunda etapa del procedimiento que demanda un tiempo adicional considerable además de paciencia para evitar la rotura de la bolsa y consecuente contaminación de la cavidad peritoneal y eventual esplenosis. En nuestro caso extrajimos el bazo indemne a través de una incisión de Pfannenstiel -donde se proyectaba el polo inferior del bazo- con el objetivo de permitir un adecuado estudio anatomopatológico del mismo y establecer un diagnóstico definitivo, además de reducir el tiempo operatorio evitando la colocación del bazo en una bolsa en un campo operatorio reducido como consecuencia de la esplenomegalia. Para evitar implantes celulares sobre la herida durante la extracción se utilizó un retractor y protector.

\section{Abstract}

Introduction: despite the fact that laparoscopic splenectomy for massive and supramassive splenomegaly constitutes a technical challenge, it is a feasible and safe procedure in the context of institutions with experienced teams in laparoscopic surgery.

Objective: to present the first case of laparoscopic splenectomy for massive splenomegaly in Uruguay.

Clinical case: the study presents the case of a 70 -year-old patient carrier of peripheral pancytopenia, 
massive splenomegaly and a diagnosis of type B lymphoproliferative neoplasm based on bone marrow aspiration and biopsy, who underwent diagnostic and therapeutic splenectomy.

The patient was operated in supine position with a 15-degree tilt, the trocars were placed under direct view, adapted to the size of the spleen which went from the diaphragm until the superior pelvic outlet. Splenectomy was performed in 220 minutes, the entire piece was removed through a hemi Pfannenstiel incision, without placing it in a bag, the wall being protected with a surgical wound retractor. There were no complications and the patient was discharged from hospital 48 hours. The blood count performed after 24 hours evidenced increase in all cell series and the pathology report confirmed diagnosis of marginal zone non- Hodgkin lymphoma.

Discussion: laparoscoppic splenectomy in massive splenomegaly requires of a greater surgical time, although blood loss and hospital star are lower when compared to conventional procedures and evidence similar morbility. The initial experience of surgical teams reports $30 \%$ of conversions and readmissions.

\section{Resumo.}

Introdução: embora a esplenectomia laparoscópica em esplenomegalias massivas e supremassivas seja um desafio técnico, sua realização é viável e segura em centros com equipes com experiência em cirurgia laparoscópica.

Objetivo: apresentar o primeiro caso de esplenectomia laparoscópica em esplenomegalia maciça realizada no Uruguai.

Caso clínico: paciente de 70 anos com pancitopenia periférica, esplenomegalia maciça e diagnóstico feito por punção de medula óssea de neoplasia linfoproliferativa tipo B de baixo grau, com indicação de esplenectomia para fins diagnósticos e terapêuticos.

A paciente foi operada em decúbito lateral direito a 15 graus, os trocartes foram colocados sob visão direta adaptados ao tamanho do baço que se estendia do diafragma ao estreito superior da pelve. A esplenectomia foi realizada em um tempo de 220 minutos, retirando-se toda a peça e sem colocá-la em bolsa por meio de uma hemi Pfannenstiel, protegendo a parede com afastador de ferida operatória.

Sem apresentar complicações a paciente teve alta após 48 horas. O hemograma realizado 24 horas depois da cirur- gia mostrou um aumento no número de todas as séries de células e o laudo anatomopatológico diagnosticou linfoma não Hodgkin de zona marginal.

Discussão: a esplenectomia laparoscópica nas esplenomegalias maciças requer um tempo cirúrgico maior, embora as perdas sanguíneas e a permanência hospitalar sejam menores em comparação aos procedimentos convencionais, apresentando morbidade semelhante. Na experiência inicial das equipes cirúrgicas, é relatado um percentual de conversões e readmissões próximo a 30\%.

\section{Bibliografía}

1. Habermalz B, Sauerland S, Decker G, Delaitre B, Gigot JF, Leandros E, et al. Laparoscopic splenectomy: the clinical practice guidelines of the European, Association for Endoscopic Surgery (EAES). Surg Endosc 2008; 22(4): 821-48.

2. Swanson TW, Meneghetti AT, Sampath S, Connors JM, Panton ON. Can J Surg 2011; 54(3):189-93.

3. Wu Z, Zhou J, Pankaj P, Peng B. Comparative treatment and literature review for laparoscopic splenectomy alone versus preoperative splenic artery embolization splenectomy. Surg Endosc 2012; 26(10):2758-66.

4. Grahn SW, Alvarez J 3rd, Kirkwood K. Trends in laparoscopic splenectomy for massive splenomegaly. Arch Surg 2006; 141(8):755-61.

5. Casaccia M, Sormani MP, Palombo D, Dellepiane C, Ibatici A. Laparoscopic splenectomy versus open splenectomy in massive and giant spleens: should we update the 2008 EAES guidelines?. Surg Laparosc Endosc Percutan Tech 2019; 29(3):178-81.

6. Tsamalaidze L, Stauffer JA, Permenter SL, Asbun HJ. Laparoscopic splenectomy for massive splenomegaly: does size matter? J Laparoendosc Adv Surg Tech A 2017; 27(10):1009-14.

7. Koshenkov VP, Németh ZH, Carter MS. Laparoscopic splenectomy: outcome and efficacy for massive and supramassive spleens. Am J Surg 2012; 203(4):517-22.

8. Rodríguez-Luna MR, Balagué C, Fernández-Ananín $\mathbf{S}$, Vilallonga R, Targarona Soler EM. Outcomes of laparoscopic splenectomy for treatment of splenomegaly: a systematic review and meta-analysis. World J Surg 2021; 45(2):465-79.

9. Shin RD, Lis R, Levergood NR, Brooks DC, Shoji BT, Tavakkoli A. Laparoscopic versus open splenectomy for splenomegaly: the verdict is unclear. Surg Endosc 2019; 33(4):1298-303.

Contribución de autores

Martina Tristant, ORCID 0000-0002-4684-4087. Concepción, diseño, redacción, revisión crítica.

Daniel González, ORCID 0000-0003-3916-9201. Concepción, diseño, redacción, revisión crítica. 